\title{
Revisiting the Martingale hypothesis for exchange rates*
}

\author{
Young-Sook Lee $^{a}$, Tae-Hwan Kim ${ }^{b, c}$ and Paul Newbold ${ }^{b}$ \\ ${ }^{a}$ Department of Economics, University of Wales Swansea \\ ${ }^{b}$ School of Economics, University of Nottingham \\ ${ }^{c}$ Department of Economics, Yonsei University
}

July 2004

\begin{abstract}
We consider a simple random walk process, a special case of the Martingale model, which exhibits a deterministic break in its drift term, for instance, from positive to negative. This particular example can be a plausible model for a time series on exchange rates which displays a persistent currency appreciation period followed by a long depreciation era. We demonstrate both theoretically and by simulation that when the standard variance-ratio test is applied to this process, the phenomenon of spurious rejections of the Martingale hypothesis can occur. We discuss some implication of this finding on the previously uncovered empirical evidence against the Martingale hypothesis for exchange rates. We further propose a fix to avoid such a problem.
\end{abstract}

Key words: Martingale hypothesis; Random walks; Variance ratio tests; Exchange rates; Spurious rejection

JEL classifications: C12, C22, F31

* Address for Correspondence: Tae-Hwan Kim, School of Economics, University of Nottingham, University Park, Nottingham, NG7 2RD, UK; Tel.: +44-115-951-5466; fax: +44-115-951-4159;

E-mail address: tae-hwan.kim@nottingham.ac.uk 


\section{Introduction}

Many structural models have attempted to explain the behavior of exchange rates under the floating rate regime after the Bretton Woods system. Meese and Rogoff (1983) found that a random walk model performs at least as well as various structural and time series models for exchange rates in terms of out-of-sample forecast. Meese and Rogoff considered three structural models such as the flexible-price monetary (Frenkel-Bilson) model, the sticky price monetary (Dornbush-Frankel) model and the sticky-price asset (HooperMorton) model, and two time series models: univariate and vector autoregressive models. Many subsequent studies have replicated their finding using different types of currencies and different forecasting models based on out-ofsample experiments [Diebold and Nason (1990), Diebold et al. (1994), Engel (1994), Frankel and Rose (1995) and Kilian and Taylor (2003)], thus supporting the notion that exchange rates might follow a random walk model.

However, Liu and He (1991) rejected the random walk hypothesis for three out of five currencies against the US dollar over the sample period from August 1974 to March 1989 using the variance-ratio test developed by Lo and MacKinlay (1988). Fong et al. (1997) reexamined the random walk hypothesis for the five exchange rates used by Liu and He (1991), employing several improved variants of variance-ratio tests in Hochberg (1974), Chow and Denning (1993) and Richardson and Smith (1991). It was found that the random walk hypothesis is rejected for the full sample period and the first subsample before October 1979 but is not rejected for the second subsample after October 1979. Based on the assumption that the central bank interventions can cause daily exchange rates to deviate from martingale behavior, Yilmaz (2003) conducted the variance-ratio test with the daily exchange rate from 1974 to 2001 in moving subsample windows with a fixed length of 1,000 daily observations. By plotting the variance-ratio test statistics as proposed by Hochberg (1974) and Richardson and Smith (1991) for all subsample windows of seven currencies against the US dollar, Yilmaz found that exchange rates tend to deviate from the random walk properties during the exchange market intervention by central banks, but was not able to test formally whether exchange rates follow a random walk model in the presence of structural breaks caused by the interventions.

It emerges from the previous work that (i) the random walk model appears to be the best for exchange rates in terms of out-of-sample forecast, but nonetheless (ii) the random walk model is often rejected when the varianceratio test is employed. In this paper, we attempt to resolve these apparently contradictory empirical findings. Our view is that (i) when a model successfully passes a battery of independent out-of-sample tests, it is likely to describe the true process, and (ii) apart from the fact that the variance-ratio test is inherently an in-sample test, it might be possible that researchers fail to take into account all the relevant characteristics of the process when con- 
ducting the variance-ratio test. One characteristic we will focus on in this paper is possible structural breaks in the exchange rate process.

Previous studies suggested that central bank interventions such as unexpected monetary contraction and transaction of foreign assets against domestic assets in the foreign exchange market can influence the level and variance of exchange rates, as documented in Dominguez (1998) and Dominguez and Frankel (1993). The time series behavior of exchange rates might also be affected by changes in economic fundamentals, external shocks such as oil crises, and unexpected changes in monetary and fiscal policies. To take into account a structural break of exchange rates, Liu and He (1991) and Fong et al. (1997) divided their whole sample with 743 observations into two subperiods before and after October 1979 when the Federal Reserve changed the operating procedure.

Dividing the sample into several subsamples can be a way of dealing with structural breaks. A problem in this approach is that the number of observations in some subsamples can be small. Small sample size can present two potential problems in conducting the variance-ratio test. First, it obviously leads to efficiency loss and reduces the power of the test as documented in Lo and MacKinlay (1989), Richardson and Smith (1991), Chow and Denning (1993) and Fong et al (1997). Secondly and more importantly, as demonstrated in Lo and MacKinlay (1989) and Fong et al. (1997), when the sample size is small, the sampling distribution of the variance-ratio statistic is not well approximated by the asymptotic standard normal distribution. In this paper, we propose a modification of the variance-ratio test using the full sample and at the same time taking into account structural breaks in the process.

The paper is organised as follows. In Section 2, we prove that the variance-ratio test statistics of Lo and MacKinlay (1988) do not tend to the standard normal distribution when there is a break in drift and we demonstrate that in such circumstances, the probability of rejecting the Martingale hypothesis goes to one when in fact the Martingale hypothesis is true. The same phenomenon is analysed in finite samples by simulation in Section 3 and we propose a modified variance-ratio test in Section 4. Section 5 applies both the standard variance-ratio tests of Lo and MacKinlay (1988) and our new test to the exchange rates of four currencies. We have found that the standard tests strongly reject the Martingale hypothesis while our tests do not. Our empirical findings strongly indicate that rejecting the Martingale hypothesis by the standard variance-ratio tests might have been induced by failing to incorporate structural breaks into the testing procedure. Finally, Section 6 provides a summary of the paper. 


\section{Spurious rejections of the Martingale hypothesis by variance-ratio tests}

We consider the following process:

$$
X_{t}=\mu_{t}+X_{t-1}+\varepsilon_{t} \quad ; \quad t=1,2, \ldots, T,
$$

or

$$
r_{t}=\Delta X_{t}=\mu_{t}+\varepsilon_{t} \quad ; \quad \Delta X_{t} \equiv X_{t}-X_{t-1} .
$$

The drift term is specified as

$$
\mu_{t}=\mu_{1} 1(t \leq[\tau T])+\mu_{2} 1([\tau T]<t),
$$

where $1(\cdot)$ is the indicator function and $[\tau T]$ is the integer part of $\tau T$ and $\tau \in(0,1)$. Whenever there is no confusion, we will use $\tau T$ in place of $[\tau T]$ from now on. The disturbance term $\varepsilon_{t}$ is assumed to satisfy that $E\left(\varepsilon_{t}\right)=0$ and $E\left(\varepsilon_{t}^{2}\right)=\sigma^{2}$. Under the Martingale hypothesis, $r_{t}$ is not serially correlated at all leads and lags; that is, $E\left(\varepsilon_{t} \varepsilon_{t-s}\right)=0$ for $t \neq s$. We also assume for convenience that $X_{0}$ is observed. The specification of the process in (1) and (2) for testing the Martingale hypothesis has been used in the previous research. The only difference is that we allow a deterministic break in the drift term.

Using the fact that the variance of the $q$-period return, $X_{t}-X_{t-q}$, is equal to $q$ times the variance of the one-period return, $X_{t}-X_{t-1}$, under the Martingale hypothesis, Lo and MacKinaly (1988) developed the varianceratio test. The $q$-period return, $r_{t}(q)$, is defined using overlapping observations:

$$
r_{t}(q)=\sum_{i=0}^{q-1} r_{t-i}=X_{t}-X_{t-q} .
$$

Variance ratio statistics could be also based on non-overlapping returns, but, as shown in Lo and MacKinlay (1988), using overlapping observations results in a more efficient test. Hence, we only consider variance-ratio statistics computed using overlapping observations. The test statistic is based on

$$
M(q)=\frac{\hat{\sigma}_{q}^{2}}{\hat{\sigma}^{2}}-1,
$$

where

$$
\begin{aligned}
\hat{\sigma}^{2} & =\frac{1}{T-1} \sum_{t=1}^{T}\left(r_{t}-\hat{\mu}\right)^{2}, \quad \hat{\mu}=\frac{1}{T} \sum_{t=1}^{T} r_{t} \\
\hat{\sigma}_{q}^{2} & =\frac{T}{q(T-q+1)(T-q)} \sum_{t=q}^{T}\left\{r_{t}(q)-q \hat{\mu}\right\}^{2} .
\end{aligned}
$$


Lo and MacKinlay (1988) proved under the Martingale hypothesis without a break in drift $\left(\mu_{1}=\mu_{2}\right)$ that (i) $M(q) \stackrel{p}{\rightarrow} 0$ and (ii) the standardized variance-ratio statistic $z(q)=T^{1 / 2}\left(\frac{2(2 q-1)(q-1)}{3 q}\right)^{-1 / 2} M(q)$ converges in distribution to $N(0,1)$. Hence, given a specific value of $q$, appropriate critical values can be chosen from $N(0,1)$ to ensure the asymptotically correct size of the test. However, the following theorem demonstrates that it is not possible to control the test size if there is a break in drift $\left(\mu_{1} \neq \mu_{2}\right)$.

Theorem 1 Suppose that $X_{t}$ is generated by (1) under the Martingale hypothesis. Then,

$$
M(q) \stackrel{p}{\rightarrow} \frac{q \delta^{2} \tau(1-\tau)}{1+\delta^{2} \tau(1-\tau)},
$$

where $\delta=\sigma^{-1}\left|\mu_{1}-\mu_{2}\right|$.

We first note that the probability limit of $M(q)$ is positive and depends on three parameters; the standardized break size $\delta$, the break time $\tau$, and the holding period $q$. It is obvious from the expression that when there is no break (either $\delta=0$ or $\tau=0,1$ ), the probability limit collapses to zero, which corresponds to the standard result. An immediate consequence of Theorem 1 is that the standardized variance-ratio statistic $z(q)$ diverges to infinity at the rate of $T^{1 / 2}$; that is, $T^{-1 / 2} z(q)=O_{p}(1)$. Thus, a routine application of the statistic $z(q)$ based on critical values from $N(0,1)$ is likely to result in spurious rejections of the Martingale hypothesis even though the null is true. As noted before, the severity of spurious rejections would depend on three parameters; $\delta, \tau$, and $q$. A closer examination of the probability limit of $M(q)$ predicts that the phenomenon of spurious rejections would be more pronounced when (i) the holding period $q$ increases, (ii) the standardized break size $\delta$ becomes larger and (iii) the structural break occurs in the middle of the sample size $(\tau=0.5)$. These predictions are confirmed in Figure 1 in which we graph the probability limit against $\delta$ and $\tau$ while fixing the holding period $q$ at two. The graph clearly shows that the probability limit is an increasing function of $\delta$ and is maximised when $\tau=0.5$.

\section{Monte Carlo simulations}

In the previous section, the phenomenon of spurious rejections has been demonstrated in large samples. Naturally, it may be interesting to investigate whether the same phenomenon can occur in finite samples and, if any, how serious the size distortion might be in such circumstances. We generate data through (1) and (2). The error terms $\varepsilon_{t}$ are drawn from $N(0,1)$. We normalise $\mu_{1}$ at zero and use various values of $\mu_{2}: \mu_{2}=0.1,0.2,0.3,0.4$. Since $\sigma=1$ and $\mu_{1}=0$, the standarised break size $\delta$ is now equal to $\mu_{2}$. In 
the simulations, we set $T=500,1000$ and $q=4,8$. The number of replications in all experiments is 1000 . In Figure 2, for the specified values of $T$ and $q$, we plot the rejection probability of the test $z(q)$ at the $5 \%$ significance level against the break fraction $\tau$ ranging from 0 to 1 . Figure 2(i) displays the results when $T=500$ and $q=4$, which indicates that the size distortion is fairly mild for smaller values of $\mu_{2}$. However, when the break fraction is around 0.5 and the standardized break size becomes larger, the rejection probability can reach up to $30 \%$. As we increase the holding period $q$ from 4 to 8 [Figure 2(ii)], and as we increase the sample size $T$ from 500 to 1000 [Figure 2(iii) and Figure 2(iv)], the size distortion becomes more pronounced. Obviously, the size distortion disappears as either $\tau \rightarrow 0$ or $\tau \rightarrow 1$ in all figures.

In Figure 2, we have investigated only the case in which there is an increase in drift; i.e. $\mu_{1}<\mu_{2}$. We have simulated the opposite cases (a decrease in drift) by setting $\mu_{2}=-0.1,-0.2,-0.3,-0.4$. The results, as expected from Theorem 1 , are entirely symmetric and the plots are identical to the ones in Figure 2. Hence, we do not report them.

Lo and MacKinlay (1988) also proposed a variance-ratio test that is robust to general forms of heteroscedasticity using the heteroscedasticityconsistent results of White $(1980,1984)$ and White and Domowitz(1984). The heteroscedasticity-robust variance-ratio test statistic, denoted by $z^{*}(q)$, is given by

$$
z^{*}(q)=\hat{V}^{-1 / 2} M(q),
$$

where

$$
\begin{aligned}
\hat{V} & =\sum_{j=1}^{q-1}\left[\frac{2(q-j)}{q}\right]^{2} \hat{\delta}(j), \\
\hat{\delta}(j) & =\frac{\sum_{k=j+1}^{T}\left(r_{k}-\hat{\mu}\right)^{2}\left(r_{k-j}-\hat{\mu}\right)^{2}}{\left[\sum_{k=1}^{T}\left(r_{k}-\hat{\mu}\right)^{2}\right]^{2}} .
\end{aligned}
$$

Under the null hypothesis that returns are heteroscedastic but uncorrelated, Lo and MacKinlay showed that $z^{*}(q)$ is asymptotically distributed as $N(0,1)$. We have repeated the same experiments as shown in Figure 2, but replacing $z(q)$ with $z^{*}(q)$. We have found that the results are virtually the same; that is, the same phenomenon of spurious rejections occur and the magnitude of spurious rejections are identical for both $z(q)$ and $z^{*}(q)$. For this reason, the results are not reported in this paper. Hence, using the heteroscedasticity-robust variance-ratio test cannot provide any protection against the size distortion problem when there is a break in drift. 


\section{Modified variance-ratio tests}

In many applications using exchange rates, it is usually assumed that structural break points are a priori known. For example, see Liu and He (1991) and Fong et al. (1997). Assuming that the break fraction $\tau$ is known, the spurious rejection problem described in the previous sections can be fixed by slightly modifying the detrending procedure in the original variance-ratio tests. Instead of demeaning the series $r_{t}$ using the whole sample, we demean $r_{t}$ in each of the subsamples. This can be done by a simple regression. We consider the following regression in which we regress $r_{t}$ on a constant and a dummy variable $d_{t}$ defined as $d_{t}=1[\tau T<t]$

$$
r_{t}=\hat{\beta}_{0}+\hat{\beta}_{1} d_{t}+\tilde{r}_{t},
$$

where $\tilde{r}_{t}$ is the residuals from the above LS regression. Our modified varianceratio statistic denoted $z_{m}(q)$ is now calculated based on $\tilde{r}_{t}$ as follows ${ }^{1}$ :

$$
z_{m}(q)=T^{1 / 2}\left(\frac{2(2 q-1)(q-1)}{3 q}\right)^{-1 / 2} M_{m}(q)
$$

where

$$
\begin{aligned}
M_{m}(q) & =\frac{\hat{\sigma}_{q m}^{2}}{\hat{\sigma}_{m}^{2}}-1, \\
\hat{\sigma}_{m}^{2} & =\frac{1}{T-1} \sum_{t=1}^{T} \tilde{r}_{t}^{2}, \\
\hat{\sigma}_{q m}^{2} & =\frac{T}{q(T-q+1)(T-q)} \sum_{t=q}^{T}\left(\sum_{i=0}^{q-1} \tilde{r}_{t-i}\right)^{2} .
\end{aligned}
$$

Using the fact that $\hat{\beta}_{0} \stackrel{p}{\rightarrow} \mu_{1}$ and $\hat{\beta}_{1} \stackrel{p}{\rightarrow} \mu_{2}-\mu_{1}$, it is straightforward to show that $z_{m}(q)$ converges in distribution to $N(0,1)$. Moreover, the same modification can be used to robustify the heteroscedasticity-robust varianceratio test in (3). We have repeated the same Monte Carlo experiments as shown in Figure 2, but replacing $z(q)$ with the new test $z_{m}(q)$. The results are displayed in Figure 3 for the specified values of $T$ and $q$, and it is clearly demonstrated that the spurious rejection phenomenon has now disappeared.

We note that our regression-based procedure can easily be extended to a situation in which there are multiple breaks as long as the break points are assumed known. For example, suppose that there are three breaks points denoted $\tau_{1}, \tau_{2}$ and $\tau_{3}$. In this case, we run the following regression:

$$
r_{t}=\hat{\beta}_{0}+\hat{\beta}_{1} d_{1 t}+\hat{\beta}_{2} d_{2 t}+\hat{\beta}_{3} d_{3 t}+\tilde{r}_{t}
$$

\footnotetext{
${ }^{1}$ The subscript $m$ indicates that the test is a modified version of the corresponding variance-ratio test.
} 
where $d_{1 t}=1\left[\tau_{1} T<t \leq \tau_{2} T-1\right], d_{2 t}=1\left[\tau_{2} T<t \leq \tau_{3} T-1\right]$ and $d_{3 t}=$ $1\left[\tau_{3} T<t\right]$. The correctly modified variance-ratio test is now obtained using the new residuals $\tilde{r}_{t}$ from the above regression (5) in the formula in (4).

\section{Re-testing the Martingale hypothesis for exchange rates}

In this section, we apply our modified variance-ratio testing procedure to revisit the Martingale hypothesis for exchange rates. Since many countries shifted to the floating exchange system during the period between 1970 and 1973, our data set starts on January 2, 1974. For the empirical study, we use weekly exchange rates for the following four currencies; Canadian dollar (CAN), German mark (DM), Italian lira (ITL), and Switzerland franc (SZF). The reason for using exchange rates from these four countries is that (i) the preliminary application of the standardised heteroscedasticity-robust variance-ratio test $z^{*}(q)$ indicates that the Martingale hypothesis is rejected for these countries and (ii) the exchange rate data from these countries clearly show that there are three distinctive structural breaks.

The Canadian dollar and the Switzerland franc end on October 15, 2003. Upon the introduction of the euro on January 1, 1999, data only extends to December 30, 1998 for German mark and Italian lira participating in the European Economic and Monetary Union. Exchange rates used in our study are the noon buying rates in New York for cable transfers payable in foreign currencies. All exchange rates are measured in the unit of foreign currency per US dollar and can be downloaded from the Federal Reserve board's website (http://www.federalreserve.gov/releases/h10/Hist). We denote by $S_{t}$ Wednesday's exchange rates and the corresponding weekly returns $r_{t}$ are calculated through $r_{t}=\ln S_{t}-\ln S_{t-1}$. If Wednesday's exchange rate is missing due to a holiday, Thursday's exchange rate (or Tuesday's if Thursday's is missing) is used because holidays occur least on Wednesdays and Thursdays. There are 1555 weekly observations for CAN and SZF and 1304 for DM and ITL.

Figure 4 provides the time series plots of the weekly exchange rates of four countries during the post-Bretton-Woods system of flexible exchange rates. As clearly indicated in the figures, there might exist three structural break points (denoted $\tau_{1}, \tau_{2}$ and $\tau_{3}$ ). Germany and Italy have common structural break points: January $1980\left(\tau_{1}\right)$, March $1985\left(\tau_{2}\right)$ and January $1988\left(\tau_{3}\right)$. The German mark and the Italian lira generally tend to move together from November 1979 when Germany and Italy joined Exchange Rate Mechanism (ERM) under European Monetary System except for the period between September 1992 and November 1996. This later disparity may have been due to the fact that Italy seceded from ERM in September 1992 and reentered in November 1996. The SZF has also three break points: 
October $1978\left(\tau_{1}\right)$, March1985 $\left(\tau_{2}\right)$ and January $1988\left(\tau_{3}\right)$, thus sharing the same two break points as in both Germany and Italy.

For these three countries, the first break picks up the start of their depreciation against the US\$ in 1980 (or at the end of the 1970s). The combination of high interest rates and low inflation rate in the US in that time period caused the dollar to appreciate against most currencies. The real interest rate in the US exhibited a sudden increase around the year 1980, which was associated with a slight delay with the change in Federal Reserve's monetary control procedure in October 1979. The three exchange rates continued to weaken throughout the early 1980s and reached all-time highs in early 1985 . The second break indicates the switch from the depreciation era to the appreciation period in March 1985. The dollar's value declined persistently from 1985 to the end of 1987, mainly due to the large trade deficit in the US during that period. The third break point denotes the end of the long appreciation era in January 1988, which was caused by reduced US trade deficit and high US interest rates.

Most currencies against the US dollar move similarly as described above and these structural break points are consistent with the dates chosen by Obstfeld (1989) and Dominguez and Frankel (1993). The Canadian dollar exhibits a quite different pattern from the other three European currencies. The average value of the changes in the CAN and its standard deviation are smaller in the absolute value than other three currencies. Unlike other European currencies, the CAN does not show a structural break in the early 1980s as chosen by Liu and He (1991). Nonetheless, the CAN currency also appears to have three structural breaks: February $1986\left(\tau_{1}\right)$, November 1991 $\left(\tau_{2}\right)$ and November $2002\left(\tau_{3}\right)$, as indicated in Figure 4(i).

After identifying the possible break points, we have applied a testing procedure to check if there is statistical evidence to justify the choice. With three break points, we have four possible drift terms denoted $\mu_{1}, \mu_{2}, \mu_{3}$ and $\mu_{4}$. Each term is estimated by

$$
\hat{\mu}_{i}=\frac{1}{\left[\tau_{i} T\right]-\left[\tau_{i-1} T\right]} \sum_{t=\left[\tau_{i-1} T\right]+1}^{\left[\tau_{i} T\right]} r_{t},
$$

where $r_{t}=\ln S_{t}-\ln S_{t-1}, \tau_{0}=0$ and $\tau_{4}=1$. Table 1 (a) presents the estimates of $\hat{\mu}_{i}$ for each sub-period and for each country. It also reports the sample standard deviations (denoted $\hat{s}_{i}$ ) of $r_{t}$ for each case. The estimates of $\hat{\mu}_{i}$ are significantly different over subsamples and the changing sign of $\hat{\mu}_{i}$ is entirely consistent with our previous discussion of the appreciation and depreciation periods; that is, the sign of the sample mean is positive (negative) when the exchange rate persistently depreciates (appreciates), which is equivalent to having a positive (negative) drift term. Having made such an observation, we have tested the null hypothesis $H_{0}: \mu_{i}=\mu_{i+1}(i=$ $1,2,3)$ for each country using a two-sample heteroscedasticity $t$-test. The 
null is rejected for all sub-periods and for all countries at the $1 \%$ significance level. The $p$-values are reported in Table 1(b).

Lo and MacKinlay (1989) examined the size and power properties of the standard variance-ratio tests for the Martingale hypothesis via Monte Carlo simulations and found that the empirical size of the variance-ratio test is close to its nominal value under the null hypothesis with independent and identically distributed Gaussian errors as well as with heteroscedastic increments for sample sizes of greater than 32. But, when the holding period $q$ increases relative to the sample size, it was also found that the sampling distribution of the standard variance-ratio tests significantly deviates from the asymptotic standard normal distribution. Fong et al. (1997) also conducted a simulation study, suggesting a reasonable range of $q$ for a given value of $T$. They used the Komogorov-Smirnov D test for normality and found that the null hypothesis of normality cannot be rejected at the $1 \%$ significance level for values of q up to 16 when the sample size is 743 . Taking into account these results in the previous research, we take a conservative approach by using $q=3,4,8$ and 16 , given that we have 1554 observations for CAN and SZF and 1303 observations for DM and ITL.

First, we apply the $z(q)$ test to the four exchange rates at $10 \%$ and $5 \%$ significance levels, assuming that there is no heteroscedasticity in the error term. The testing results are displayed in Table 2. The evidence from the data is pointing strongly against the Martingale hypothesis; the null is rejected for $q=3,4$ in CAN, for $q=3,4,16$ in SZF, and for all values of $q$ in both DM and ITL. Next, we apply our modified version $z_{m}(q)$, calculated through the auxiliary LS regression in (5), in which the exact dates for $\tau_{1}, \tau_{2}$ and $\tau_{3}$ have been already specified above. The results are also in Table 2, which shows that (i) all the modified test statistics become much smaller than the unmodified ones and (ii) we fail to reject the Martingale hypothesis in all series but CAN. Even in that case, we reject the null at the $10 \%$ significance level, only when $q=3$.

The strong evidence against the Martingale hypothesis by the $z(q)$ test might have been caused by the possible presence of heteroscedasticity in the considered exchange rates. Hence, we also apply the heteroscedasticityconsistent variance-ratio test statistic $z^{*}(q)$ whose results are in Table 3. Table 3 indicates that heteroscedasticity must have played a role because we have now a much smaller number of rejections of the Martingale hypothesis, compared to the $z(q)$ test; the null is rejected for $q=3$ in CAN, for $q=8,16$ in SZF, for $q=8,16$ in ITL, and for all values of $q$ in DM. The evidence has been much weakened, but we still reject the null for all countries. Finally, we apply our modified $z_{m}^{*}(q)$ test. Table 3 shows that the Martingale hypothesis is not rejected in any cases. 


\section{Summary}

This research has been mainly motivated by the apparentry contradictory empirical findings in the literature on the role of the Martingale hypothesis in formulating the correct model for exchange rates; some evidence supporting the hypothesis has been put forward while the opposite evidence has surfaced via the variance-ratio test. We have demonstrated that, if a break in drift is not properly taken into account, then the routine application of the variance-ratio test can result in potentially large size distortion; rejecting the Martingale hypothesis $100 \%$ of the time asymptotically when the process is actually a Martingale. Based on a simple regression approach, we have proposed a modification of the test such that the size of the test is correctly controlled. When the standard variance-ratio tests and our modified tests are applied to exchange rates of CAN, DM, ITL and SZF, the test results clearly show that the rejection of the Martingale hypothesis by the standard variance-ratio tests may have been caused by ignoring the presence of structural breaks.

\section{Appendix: Proof of Theorem 1}

Recalling that $M_{r}(q)=\hat{\sigma}^{-2} \hat{\sigma}_{q}^{2}-1$, we first show examine the probability limit of $\hat{\sigma}^{2}$ :

$$
\begin{aligned}
\hat{\sigma}^{2}= & T^{-1} \sum_{t=1}^{T}\left(r_{t}-\widehat{\mu}\right)^{2}+o_{p}(1) \\
= & \tau \mu_{1}^{2}+(1-\tau) \mu_{2}^{2}+T^{-1} \sum_{t=1}^{T} \varepsilon_{t}-\widehat{\mu}^{2} \\
& \stackrel{p}{\rightarrow} \sigma^{2}\left\{1+\delta^{2} \tau(1-\tau)\right\}
\end{aligned}
$$

using that fact that

$$
\widehat{\mu} \stackrel{p}{\rightarrow} \tau \mu_{1}+(1-\tau) \mu_{2} .
$$

Next, we turn to the variance estimator based on $q$-period returns

$$
\begin{aligned}
\hat{\sigma}_{q}^{2} & =(q T)^{-1} \sum_{t=q}^{T}\left(r_{t}(q)-q \widehat{\mu}\right)^{2}+o_{p}(1) \\
& =q^{-1} \sum_{i=0}^{q-1} T^{-1} \sum_{t=q}^{T}\left(r_{t-i}-\widehat{\mu}\right)^{2}+q^{-1} \sum_{i=0}^{q-1} \sum_{j=0(\neq i)}^{q-1} T^{-1} \sum_{t=q}^{T}\left(r_{t-i}-\widehat{\mu}\right)\left(r_{t-j}-\widehat{\mu}\right) .
\end{aligned}
$$

It is straightforward to show that

$$
T^{-1} \sum_{t=q}^{T}\left(r_{t-i}-\widehat{\mu}\right)^{2} \stackrel{p}{\rightarrow} \sigma^{2}\left\{1+\delta^{2} \tau(1-\tau)\right\}
$$




$$
T^{-1} \sum_{t=q}^{T}\left(r_{t-i}-\widehat{\mu}\right)\left(r_{t-j}-\widehat{\mu}\right) \stackrel{p}{\rightarrow} \sigma^{2} \delta^{2} \tau(1-\tau) .
$$

Hence, we have

$$
\hat{\sigma}_{q}^{2} \stackrel{p}{\rightarrow} \sigma^{2}\left\{1+q \delta^{2} \tau(1-\tau)\right\} .
$$

Combining the results in (6) and (7), we obtain the desired result:

$$
M(q) \stackrel{p}{\rightarrow} \frac{q \delta^{2} \tau(1-\tau)}{1+\delta^{2} \tau(1-\tau)}
$$

where $\delta=\sigma^{-1}\left|\mu_{1}-\mu_{2}\right|$.

\section{References}

[1] Chow, K.V., Denning, K.C., 1993. A simple multiple variance test. Journal of Econometrics 59, 385-401.

[2] Diebold, F.X., Garneazabal, J., and Yilmaz, K., 1994. On cointegration and exchange rate dynamics. Journal of Finance 49, 727-735.

[3] Diebold, F.X., Nason, J.A., 1990. Nonparmetric exchange rate prediction? Journal of International Economics 28, 315-332.

[4] Dominguez, K, 1998. Central bank intervention and exchange rate volatility. Journal of International Money and Finance 17, 161-190.

[5] Dominguez, K., Frankel, J., 1993. Does foreign exchange intervention matter?: the portfolio effect. American Economic Review 83, 1356-69.

[6] Engel, C., 1994. Can Markov switching model forecast exchange rates? Journal of International Economics 36, 151-165.

[7] Fong, W.M., Koh, S.K. and Ouliaris, S., 1997. Joint variance-ratio tests of the Martingale hypothesis for exchange rates. Journal of Business and Economic Statistics 15, 51-59.

[8] Frankel, J.A., Rose, A.,1995. Empirical research on nominal exchange rates. Handbook of International Economics.

[9] Hochberg, Y., 1974. Some generalization of T-method in simulations inference. Journal of Multivariate Analysis 4, 224-234.

[10] Kilian, L., Taylor, M.P., 2003. Why is it so difficult to beat the random walk forecast of exchange rate? Journal of International Economics 60, $85-107$. 
[11] Liu, C.Y., He, J., 1991. A variance-ratio test of random walks in foreign exchange rates. Journal of Finance 46, 773-785.

[12] Lo, A.W., MacKinlay, A.G., 1988. Stock market prices do not follow random walks: evidence from a simple specification test. Review of Financial Studies 1, 41-66.

[13] Lo, A.W., MacKinlay, A.G., 1989. The size and power of the variance ratio test in finite samples. Journal of Econometrics 40, 203-238.

[14] Meese, R.A., Rogoff, K., 1983. Empirical exchange rate models of the seventies: do they fit out of sample? Journal of International Economics $14,3-24$.

[15] Richardson, M., Smith, T. 1991. Tests of financial models in the presence of overlapping observations. Review of Financial Studies 4, 227254 .

[16] White, H., 1980. A heteroscedasticity-consistent covariance matrix estimator and a direct test for heteroscedasticity. Econometrica 48, 817838.

[17] White, H., 1984. Asymptotic Theory for Econometricians, Academic Press, New York.

[18] White, H., Domowitz, I, 1984. Nonlinear regression with dependent observations. Econometrica 52, 143-162.

[19] Yilmaz, K., 2003. Martingale property of exchange rates and central bank interventions. Journal of Business \& Economic Statistics 21, 383395. 
Table 1(a). Descriptive statistics for weekly returns

\begin{tabular}{|c|c|c|c|c|}
\hline & CAN & SZF & DM & ITL \\
\hline full sample & Jan. 2, '74- & Jan. 2, '74- & Jan. 2, '74- & Jan. 2, '74- \\
\hline period & Oct. $15,{ }^{\prime} 03$ & Oct. $15,{ }^{\prime} 03$ & Dec. 30, ' 30 & Dec. 30, '98 \\
\hline \#Obs. & 1554 & 1554 & 1303 & 1303 \\
\hline mean $\left(\hat{\mu}_{1}\right)$ & 0.0002 & -0.0006 & -0.0004 & 0.0008 \\
\hline stand. dev. $\left(\hat{s}_{1}\right)$ & 0.0063 & 0.0162 & 0.0146 & 0.0142 \\
\hline & Jan. 2, '74 - & Jan. 2, '74 - & Jan.2,'74- & Jan.2,'74- \\
\hline subperiod 1 & Feb. 5, '86 & Sep. $27, ' 78$ & Jan.9,'80 & Jan.2,'80 \\
\hline \#Obs. & 631 & 247 & 314 & 313 \\
\hline mean $\left(\hat{\mu}_{2}\right)$ & 0.0006 & -0.0032 & -0.0015 & 0.0008 \\
\hline stand. dev. $\left(\hat{s}_{2}\right)$ & 0.0054 & 0.0143 & 0.0108 & 0.0114 \\
\hline subnoriod 2 & Feb.12,'86- & Oct.4,'78- & Jan.16,'80- & Jan.9,'80- \\
\hline & Oct.30,'91 & Mar.6,'85 & Mar.6,'85 & Mar.13,'85 \\
\hline \#Obs. & 299 & 336 & 269 & 271 \\
\hline mean $\left(\hat{\mu}_{2}\right)$ & -0.0008 & 0.0020 & 0.0025 & 0.0036 \\
\hline stand. dev. $\left(\hat{s}_{3}\right)$ & 0.0056 & 0.0163 & 0.0150 & 0.0135 \\
\hline subperiod 3 & $\begin{array}{c}\text { Nov. } 6,1991- \\
\text { Oct. } 9,2002\end{array}$ & $\begin{array}{c}\text { Mar. 13, '85 - } \\
\text { Dec. 30, ' } 87\end{array}$ & $\begin{array}{c}\text { Mar. } 13, ' 85- \\
\text { Dec. } 30,{ }^{\prime} 87\end{array}$ & $\begin{array}{l}\text { Mar. 20, '85 } \\
\text { Dec. 30, ' } 87\end{array}$ \\
\hline$T_{3}$ & 571 & 147 & 147 & 146 \\
\hline mean $\left(\hat{\mu}_{3}\right)$ & 0.0006 & -0.0055 & -0.0051 & -0.0040 \\
\hline stand. dev. $\left(\hat{s}_{3}\right)$ & 0.0067 & 0.0195 & 0.0176 & 0.0161 \\
\hline subperiod 4 & $\begin{array}{c}\text { Nov. } 16, \text { '02 - } \\
\text { Oct. } 15,{ }^{\prime} 03\end{array}$ & $\begin{array}{l}\text { Jan. } 6, ' 88- \\
\text { Oct. } 15,{ }^{\prime} 03\end{array}$ & $\begin{array}{l}\text { Jan. 6, ' } 88 \text { - } \\
\text { Dec. } 30,{ }^{\prime} 98\end{array}$ & $\begin{array}{l}\text { Jan. } 6,{ }^{\prime} 88- \\
\text { Dec. } 30,{ }^{\prime} 98\end{array}$ \\
\hline \#Obs. & 53 & 824 & 573 & 573 \\
\hline mean $\left(\hat{\mu}_{4}\right)$ & -0.0035 & 0.0000 & 0.0001 & 0.0006 \\
\hline stand. dev. $\left(\hat{s}_{4}\right)$ & 0.0107 & 0.0158 & 0.0150 & 0.0150 \\
\hline
\end{tabular}

Table 1(b). P-values for two-sample heteroscedasticity $t$-tests

\begin{tabular}{ccccc}
\hline$H_{0}$ & CAN & SZF & DM & ITL \\
$\mu_{1}=\mu_{2}$ & 0.001 & 0.001 & 0.000 & 0.009 \\
$\mu_{2}=\mu_{3}$ & 0.001 & 0.001 & 0.000 & 0.000 \\
$\mu_{3}=\mu_{4}$ & 0.008 & 0.001 & 0.001 & 0.001 \\
\hline
\end{tabular}


$\underline{\text { Table 2. Variance-ratio test results using } z(q) \text { and } z_{m}(q)}$

\begin{tabular}{cccccc}
\hline \multicolumn{5}{c}{3} & \multicolumn{3}{c}{$q$} \\
\hline \multirow{2}{*}{ CAN } & $z(q)$ & $2.574^{a}$ & $1.874^{b}$ & 0.979 & 0.307 \\
& $z_{m}(q)$ & $1.708^{b}$ & 0.843 & -0.044 & -1.493 \\
\hline \multirow{2}{*}{ DM } & $z(q)$ & $2.132^{a}$ & $2.032^{a}$ & $2.067^{a}$ & $2.278^{a}$ \\
& $z_{m}(q)$ & 1.166 & 0.886 & 0.469 & 0.164 \\
\hline \multirow{2}{*}{ ITL } & $z(q)$ & 1.443 & $1.846^{b}$ & $2.560^{a}$ & $3.055^{a}$ \\
& $z_{m}(q)$ & 0.530 & 0.775 & 1.055 & 1.002 \\
\hline \multirow{2}{*}{ SZF } & $z(q)$ & $1.801^{b}$ & $1.834^{b}$ & $2.383^{a}$ & $2.561^{a}$ \\
& $z_{m}(q)$ & 0.945 & 0.815 & 0.995 & 0.841 \\
\hline
\end{tabular}

Note: The superscript a or $\mathrm{b}$ indicates that the test result is significant at the $5 \%$ or $10 \%$ significance level respectively. 
Table 3. Variance-ratio test results using $z^{*}(q)$ and $z_{m}^{*}(q)$

\begin{tabular}{cccccc}
\hline & & \multicolumn{5}{c}{$q$} \\
\hline \multirow{2}{*}{$\mathrm{CAN}$} & $z^{*}(q)$ & $1.961^{a}$ & 1.447 & 0.780 & 0.253 \\
& $z_{m}^{*}(q)$ & 1.331 & 0.665 & -0.358 & -1.412 \\
\hline \multirow{2}{*}{$\mathrm{DM}$} & $z^{*}(q)$ & $1.894^{b}$ & $1.763^{b}$ & $1.773^{b}$ & $1.997^{a}$ \\
& $z_{m}^{*}(q)$ & 1.037 & 0.766 & 0.400 & 0.143 \\
\hline \multirow{2}{*}{ ITL } & $z^{*}(q)$ & 1.150 & 1.433 & $1.949^{b}$ & $2.411^{a}$ \\
& $z_{m}^{*}(q)$ & 0.420 & 0.598 & 0.797 & 0.786 \\
\hline \multirow{2}{*}{$\mathrm{SZF}$} & $z^{*}(q)$ & 1.632 & 1.642 & $2.107^{a}$ & $2.228^{a}$ \\
& $z_{m}^{*}(q)$ & 0.855 & 0.730 & 0.882 & 0.751 \\
\hline
\end{tabular}

Note: The superscript a or b indicates that the test result is significant at the $5 \%$ or $10 \%$ significance level respectively. 


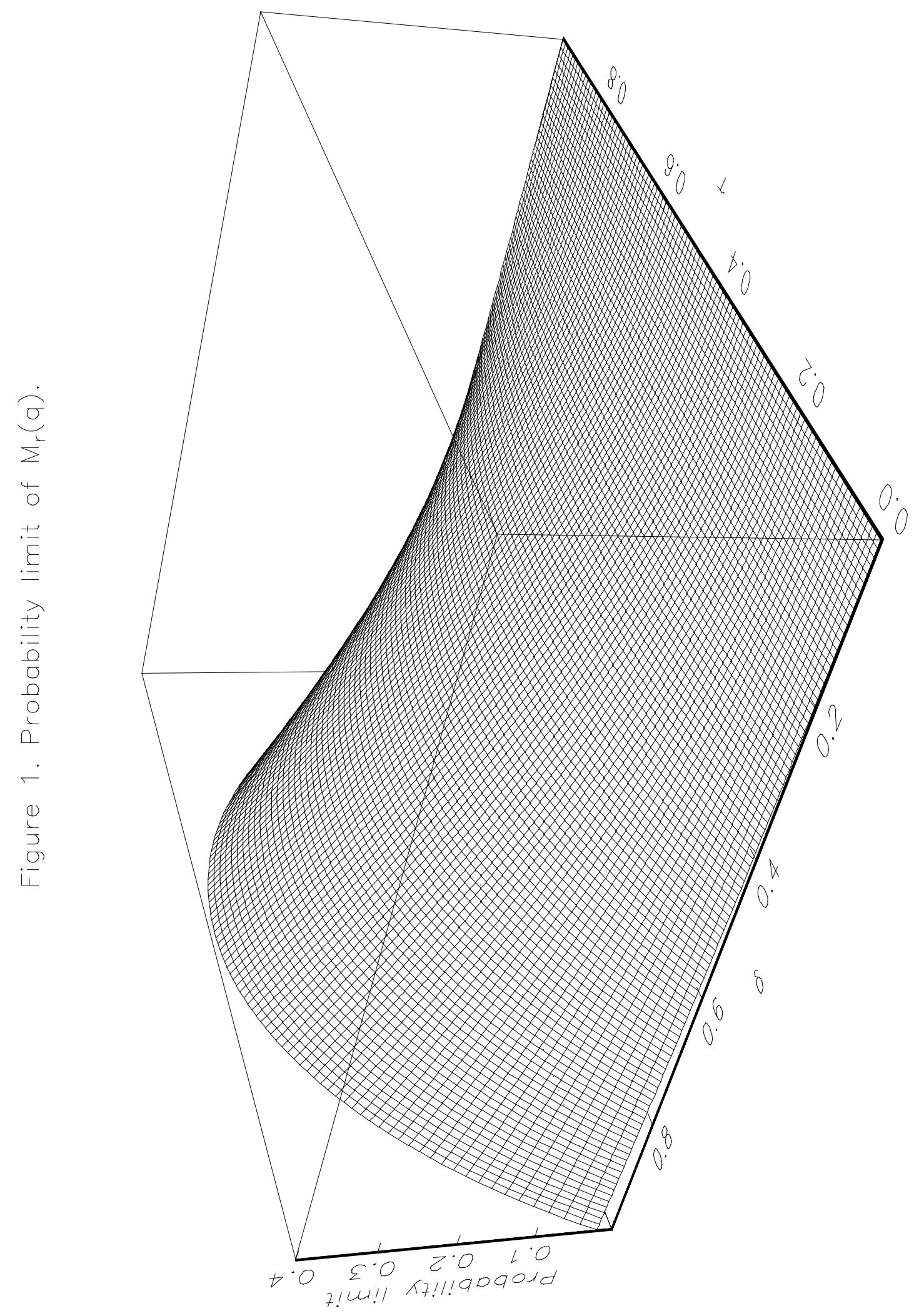



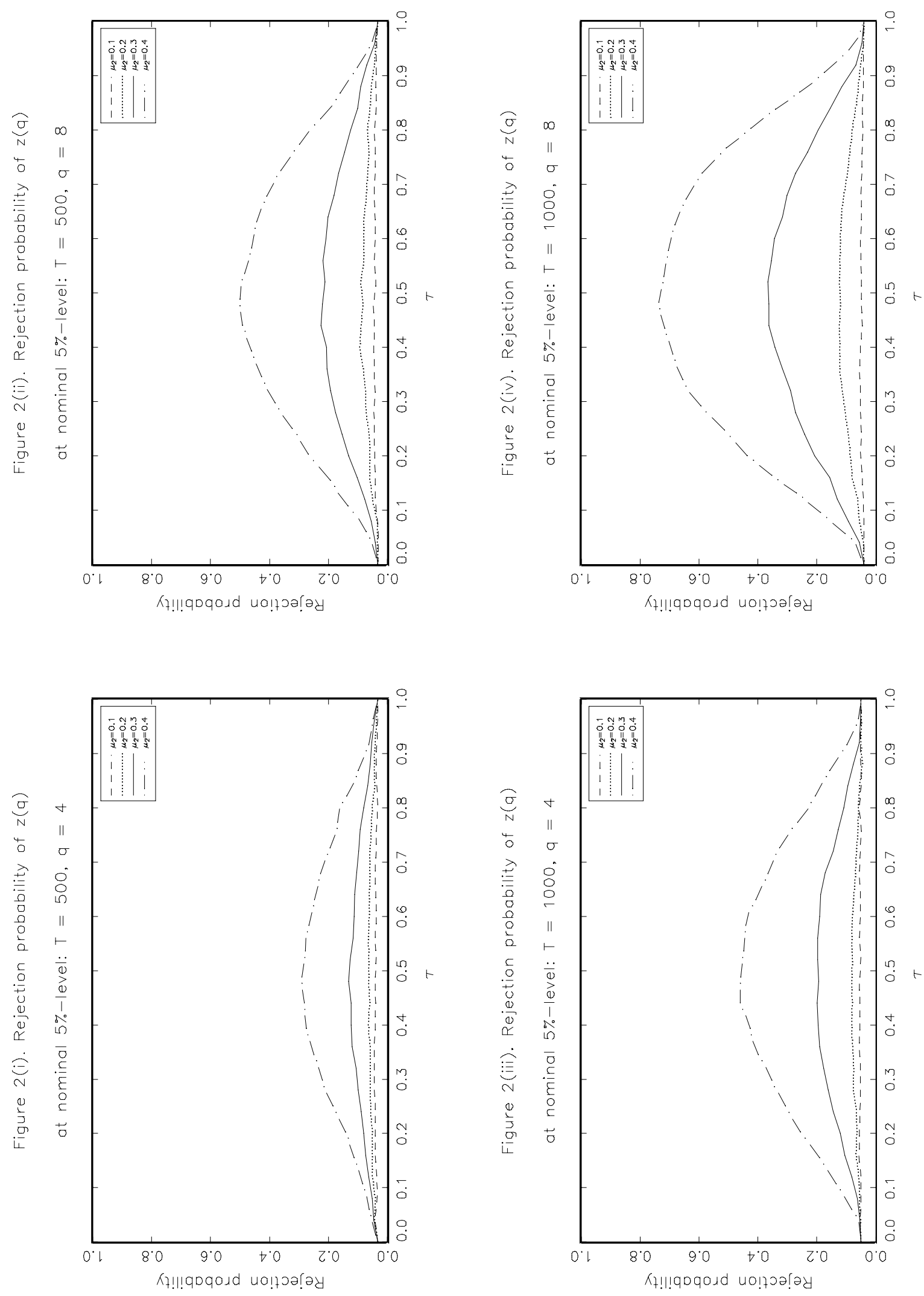

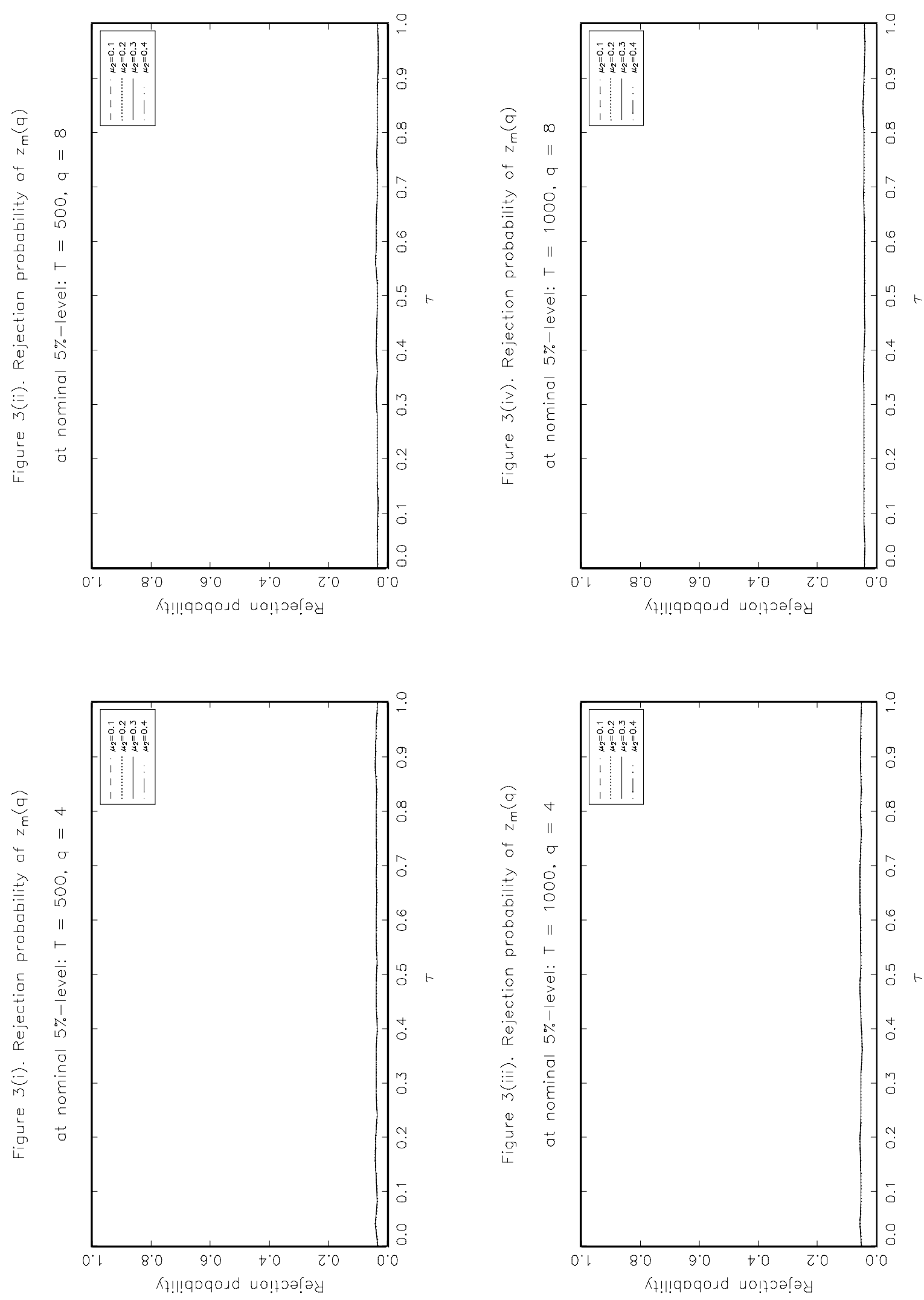

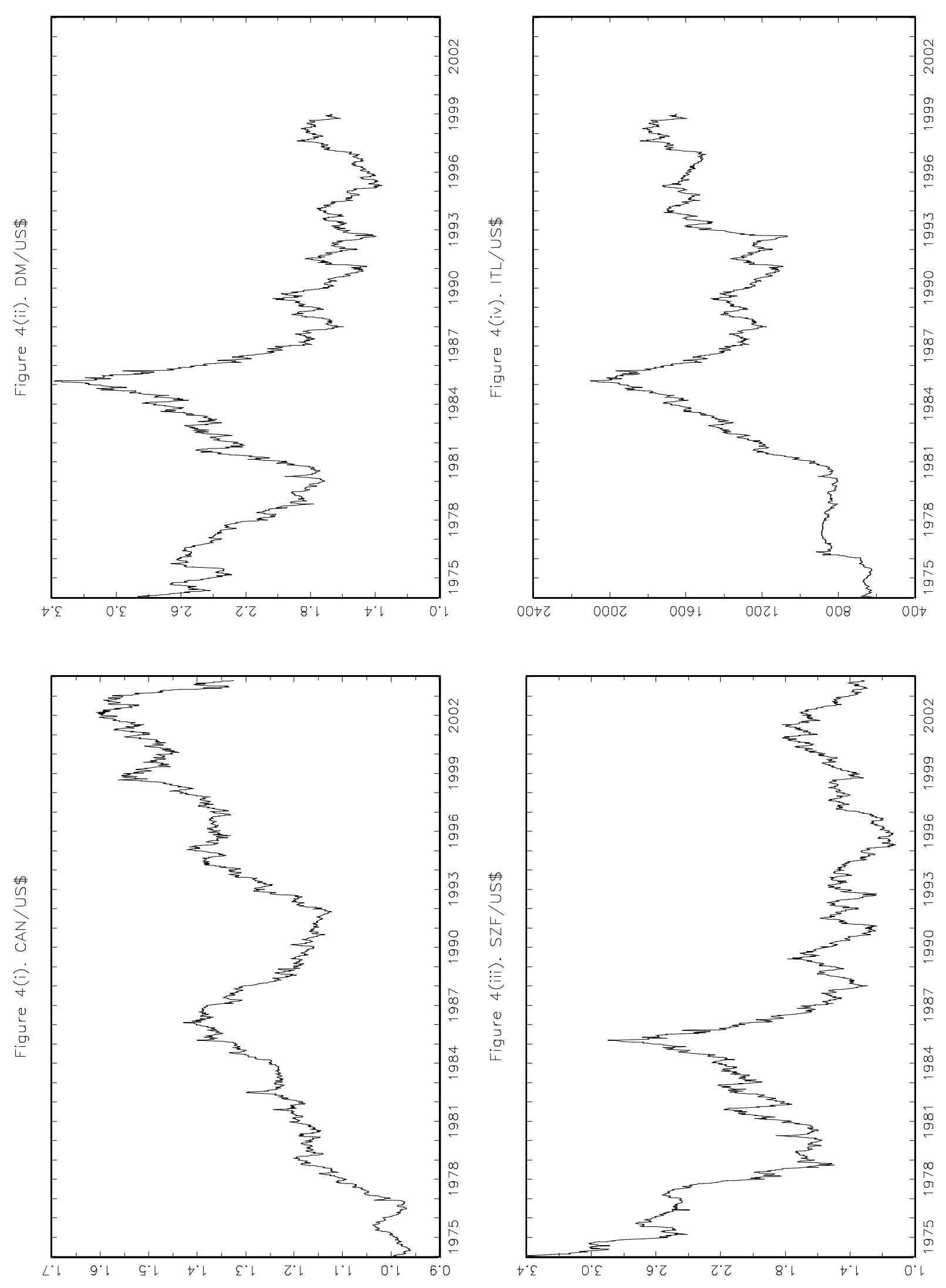